\title{
A mesocosm experiment on the effects of hydrocarbon and copper pollution on a sublittoral soft-sediment meiobenthic community
}

\author{
R. M. Warwick ${ }^{1}$, M. R. Carr ${ }^{1}$, K. R. Clarke ${ }^{1}$, J. M. Gee ${ }^{1}$, R. H. Green ${ }^{2}$ \\ ${ }^{1}$ Plymouth Marine Laboratory (West Hoe), Prospect Place, The Hoe, Plymouth PL1 3DH, United Kingdom \\ ${ }^{2}$ Department of Zoology, University of Western Ontario, London, Ontario, Canada N6A 5B7
}

\begin{abstract}
Meiofaunal assemblages in undisturbed box-core samples of sublittoral sediment were subjected to 4 dose levels of pollutants (copper and hydrocarbons) in a mesocosm experiment designed for the GEEP Workshop. Multivariate analyses of species abundance data for nematodes and copepods showed only marginal differences in species composition between treatments at the end of the experiment, principally between the high-dose treatment and the others. Diversity profiles for the nematodes were virtually unaffected, but copepods showed a graded response of decreasing diversity with increasing dose level, both intrinsically and relative to the nematode diversity profiles. Changes in copepod diversity were brought about by disproportionate increases in abundance of certain species rather than selective losses, a situation which parallels the effects of particulate organic enrichment.
\end{abstract}

\section{INTRODUCTION}

In many respects meiobenthos are much more amenable than macrobenthos to experiments in microcosms and mesocosms which are designed to investigate community-level responses to various kinds of manipulation. Firstly, because of their size and turnover time, community responses are measurable on spatial and temporal scales which can be reproduced in such experiments, whereas this is not generally the case for macrofauna. Secondly, it is often difficult to provide conditions within microcosms or mesocosms for macrobenthic species with planktonic larvae to complete their life-cycle, i.e. recruitment is precluded so that the only possible changes in community structure in pollution studies will result from selective mortality of the more sensitive species rather than increases in abundance of the less sensitive. Because meiobenthic species have direct benthic development, more realistic changes in community structure can occur, involving both mortality and recruitment.

To date, experimental manipulations of meiobenthos have largely utilised microcosms comprising either flow-through sand columns (McIntyre et al. 1970, Boucher \& Chamroux 1976, Chamroux et al. 1977, Boucher 1979, Wormald \& Stirling 1979, McLachlan et al. 1981) or small aquaria (Cantelmo \& Rao 1978, Cantelmo et al. 1979), which have all demonstrated significant effects of treatments, including additions of various levels and types of pollutants. On a larger scale, Bell \& Coull (1978) used a mesocosm set-up to investigate the importance of dagger shrimp predation on meiofauna. Elmgren et al. (1980), Grassle et al. (1980) and Elmgren \& Frithsen (1982) used the MERL mesocosm systems at Rhode Island to investigate the effects of low level additions of No. 2 fuel oil; meiofauna were only identified to major taxon level, but nevertheless significant treatment effects were observed which paralleled the effects of an oil-spill in the field. The Solbergstrand mesocosm system, where the present experiments were conducted, has been used previously to investigate the effects of different levels of particulate organic input (Gee et al. 1985) and the feeding activity of macrobenthic organisms (Warwick et al. 1986) on meiobenthic community structure.

Appropriate techniques for analysing the results of such mesocosm experiments are essentially the same as those used for the analysis of field survey data (Gray et al. 1988, Heip at al. 1988). Multivariate classification and ordination can be used to assess whether there are any significant differences in the species composition of communities between treatments, and univariate 
measures (in which the actual identity of each species is not retained in comparisons between treatments) can be used as indices of biological stress. In a properly designed mesocosm experiment, however, the third stage of correlating the results of the multivariate and univariate analyses with both natural and anthropogenic environmental variables is of course unnecessary, since all variables except the experimental one should be the same between treatments. The level of community response to measured levels of pollutants under these controlled conditions provides a test for cause and effect which can only be inferred from correlations between pollutant levels and benthic community structure in the field.

\section{METHODS}

Methods of collection of the sediment samples from Bjørnehodet Bay, and the experimental mesocosm design and pollution dosing levels, are given by Bakke et al. (1988). Four $28 \mathrm{~cm}^{2}$ sediment cores were taken at random positions within each of 4 sediment boxes from each treatment on 10 July, i.e. 12 wk after the boxes had been brought in from the field and $11 \mathrm{wk}$ after pollution dosing commenced. Core samples were preserved in $10 \%$ formalin and sent to Plymouth, UK where the meiofauna were analysed 'blind', i.e although the samples from the 4 boxes within each treatment were identified as replicates, there was no prior knowledge of the allocation of each set to a particular treatment.

In view of the large size of the samples, only a subsample of each was analysed. The sediment was suspended evenly in $500 \mathrm{ml}$ of liquid (the formalin was made up to this volume with tap water) and an $80 \mathrm{mI}$ subsample rapidly withdrawn. Subsamples from each. of the 4 replicate cores were combined to give a single sample for each box, equivalent to a surface area of $17.9 \mathrm{~cm}^{2}$. This procedure resulted in a much more representative sample of the meiofauna of the whole box than a single sample of equivalent area would have done. Each combined sample was washed in tap water on a $63 \mu \mathrm{m}$ sieve, and the meiofauna extracted by flotation in LUDOX TM colloidal silica, repeated 4 times. Copepods were then picked out from the samples under a binocular microscope, since their identification often requires dissection, and the remaining meiofauna were slowly evaporated to anhydrous glycerine and mounted evenly spread on slides for identification and counting under a high power microscope. All techniques for sample extraction and processing are detailed in McIntyre \& Warwick (1984).

Previous mesocosm experiments with meiofauna from the same field site (Gee et al. 1985, Warwick et al.
1986) had shown the meiofauna to be dominated by nematodes and copepods, as is usual for sublittoral muds (Mcintyre 1969). Our analysis has therefore been confined to these 2 taxa. In any case, for many of the soft-bodied taxa, identification to species level is not possible in formalin preserved samples. Nematodes were always much more abundant than copepods, and the first 100 specimens encountered on scanning the slides were identified to species. These counts were corrected for total abundance in the sample, which was determined from total nematode counts along optical transects across the slides. All post-naupliar copepod specimens were identified, but some copepodites could only be determined to family and these have been omitted from all numerical analyses, except for total abundance estimates

Numerical analyses performed on the species abundance data include various multivariate procedures. A similarity matrix was constructed using the Bray-Curtis measure of similarity on data subjected to various strengths of transformation; this matrix was then subjected to classification using group average sorting, to multidimensional scaling ordination (MDS), and to the significance test ANOSIM (Clarke \& Green 1988). Detrended Correspondence Analysis (DECORANA, Hill \& Gauch 1980) was also performed.

In order to carry out correlation-based Principal Component Analysis (PCA), it was necessary to reduce the data matrix. Those species representing less than $3 \%$ of the total number of individuals in any one sample were retained (26 nematode and 26 copepod species). PCA was supplemented by 'classical' ANOVA and MANOVA tests exactly as described in Gray et al. (1988), followed by pairwise comparison between treatment means based on Malhanobis' distances (Seber 1984). All analyses based on multivariate-normal distributional assumptions were performed on the widely available SAS statistics package (SAS 1985).

Univariate techniques included the calculation of the Shannon-Wiener diversity index $\left(H^{\prime}\right)$, evenness as Pielou's $J$, species richness as Margalef's $D$, Hill's diversity numbers (Hill 1973), see Heip et al. (1988), and the plotting of $k$-dominance curves (Lambshead et al. 1983). All these analyses were performed on nematodes and copepods separately, because in view of the different methods of abundance calculation it was not desirable to combine the data sets. The concept of indicator species or taxa is not so highly developed for meiofauna as for macrofauna, but we have calculated the now rather controversial nematode:copepod ratio (Raffaelli \& Mason 1981), and as far as possible have tried to interpret any species changes in terms of the 'opportunistic' or 'conservative' life-history characteristics of the species involved. 


\section{RESULTS}

The raw data on species abundances of nematodes and copepods in the 16 samples are given in Appendix 3. Tables 8 and 9 .

\section{Multivariate analyses}

Multidimensional Scaling (MDS) ordination of the nematode abundance data using various transformations and the Bray-Curtis similarity measure shows no clear separation of replicates within treatments (Fig. 1).

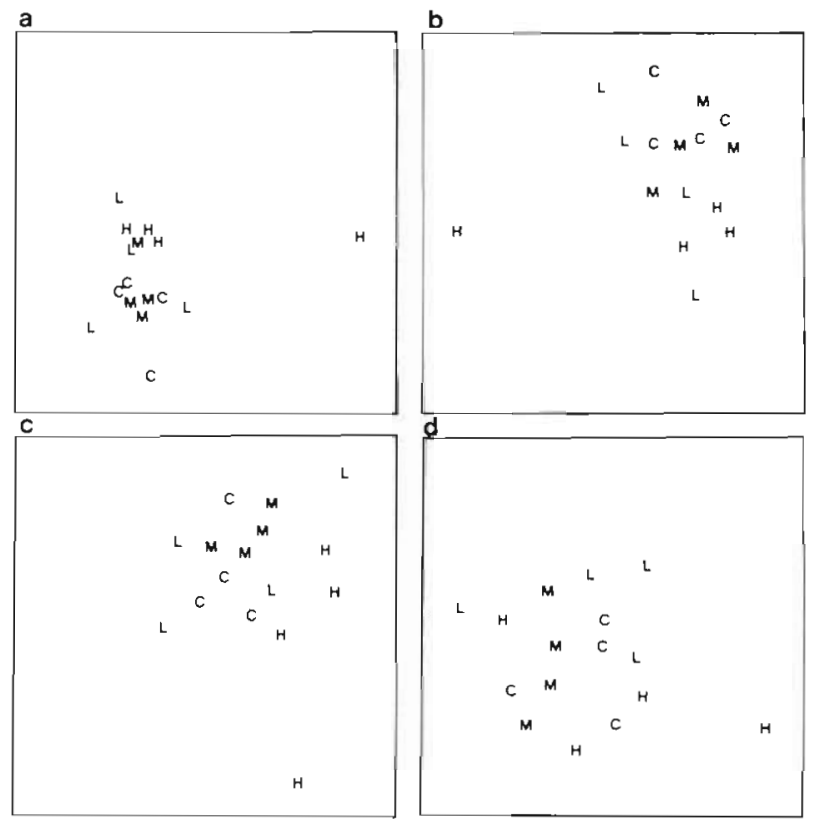

Fig. 1 MDS ordination of nematode abundance data (all species) for the 4 replicate boxes from each mesocosm treatment ( $\mathrm{C}$ : control, L: low, $\mathrm{M}$ : medium, $\mathrm{H}$ : high dose of a diesel oil/Cu mixture), based on Bray-Curtis similarities computed under various transformations. (a) No transformation, (b) , (c) $\therefore$ (d) presence/absence. Stress values are: (a) 0.08, (b) 0.14 , (c) 0.19 , (d) 0.19 . Note that plots can be arbitrarily oriented or transposed; no attempt has been made to align them

Stress values were quite high in all cases, indicating that a 2-D ordination is a mediocre representation of the data, but ordinations in 3-D (with acceptable stress levels of around 0.1) fail to show clearer separations. On all configurations, however, one of the high dose samples (the second $\mathrm{H}$ replicate in Appendix 3, Table 8 ) is clearly distinguished from the remainder. Classification using group average sorting produced dendrograms which simply confirmed the MDS results. The results of ANOSIM (Clarke \& Green 1988) for similarities from both square root and 4 th root transformations indicate that there are, however, significant differences among treatments $(p<0.05)$, and pairwise comparisons indicate that these differences are between both the high-dose treatment $(\mathrm{H})$ and the medium dose ( $M$ ) and between $\mathrm{H}$ and control $(C)$.
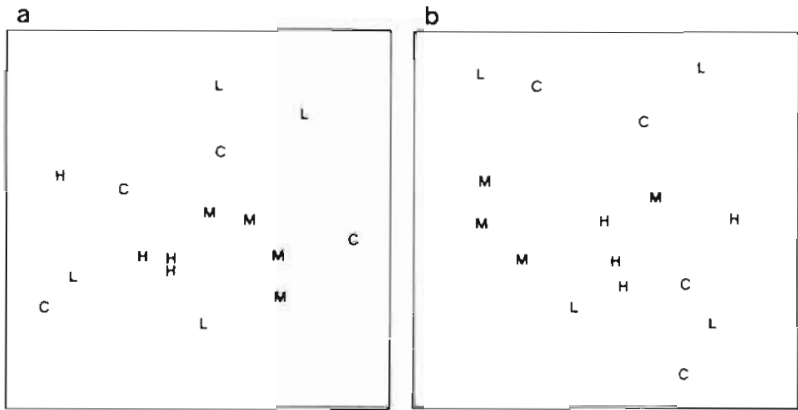

Fig. 2. MDS ordination of copepod abundance data (all species), using the Bray-Curtis similarity measure under (a) and (b) "transformations (orientation is arbitrary). Treatment notation as Fig. 1. Stress is 0.18 in both cases
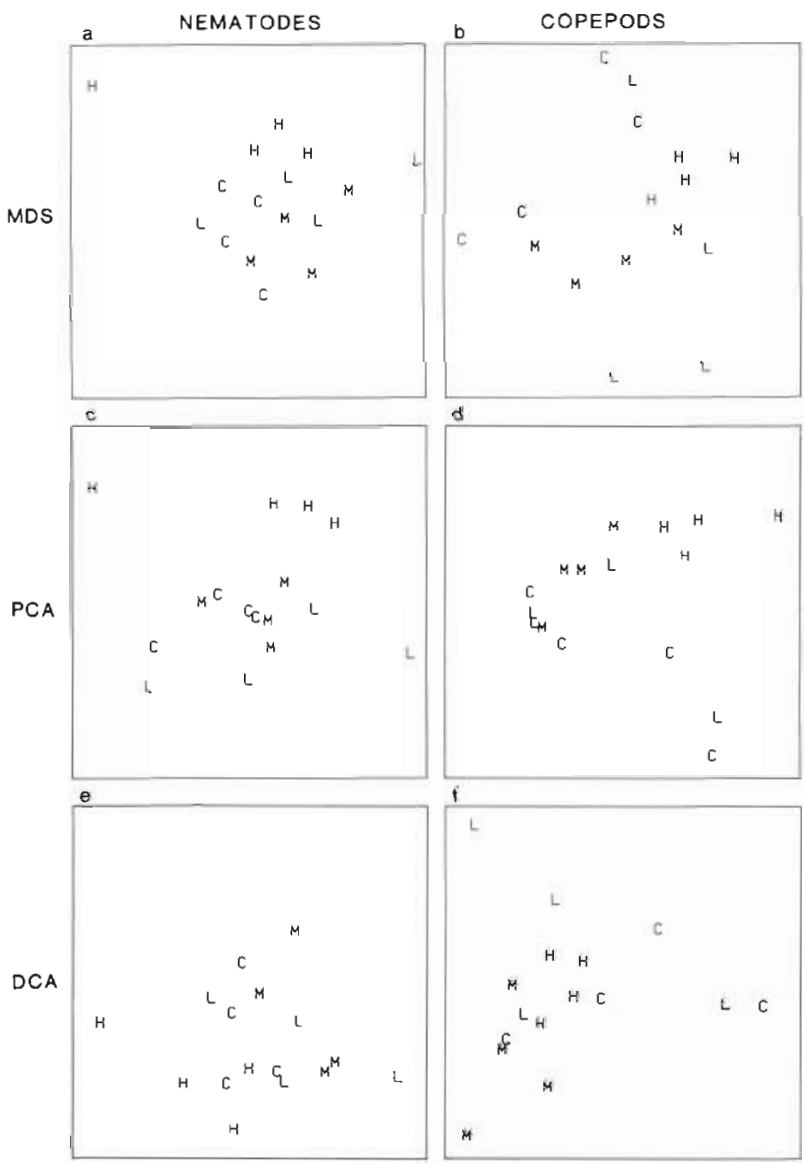

Fig. 3. Comparison of 2-D ordinations produced by MDS (arbitrary orientation), PCA (abscissa: PC1, ordinate: PC2) and DECORANA (abscissa: Axis 1, ordinate: Axis 2) for a reduced species set of nematodes and copepods (species $>3 \%$ abundance in any one replicate retained), using Iog $(1+x)$ transformed abundances. Treatment notation as Fig. 1. MDS stress values are: (a) 0.19, (b) 0.18. For PCA, the percentages of the variability explained by $\mathrm{PC} 1$ and $\mathrm{PC} 2$ are: (c) 22.5 and 14.6, (d) 23.2 and 18.2 
For copepods, MDS again shows no clear clustering of replicates within treatments (examples for square and 4 th root transformed data are given in Fig. 2.) Stress values are again high but dendrograms confirm that there is no clear pattern. ANOSIM for 4 th root transformed data shows no significant differences among treatments, but for square root transformed data there are marginally significant differences between the high-dose treatment $(\mathrm{H})$ and the medium dose (M) and control (C), and between $M$ and $C$. The effect of the transformation on the outcome of ANOSIM suggests that the differences among treatments tend to be in the more common species which are more highly weighted by the square root than 4 th root transformation.

Two further ordination methods, Principal Component Analysis (PCA) and Detrended Correspondence Analysis (DECORANA), are contrasted with an MDS configuration in Fig. 3, for both nematodes and copepods. The comparison presented here is restricted to a single transformation, that of $\log (1+x)$; this produces results that are similar to a 4 th root transform, for both MDS and PCA. Covariance-based PCA was also performed, with broadly similar results. Note that, in order to ensure comparability, the MDS and DECORANA analyses were performed on the restricted subset of species used for the PCA. MDS does not normally require this reduction since, unlike a correlation coefficient, a Bray-Curtis similarity automatically downweights the effect of rare species, and eliminates altogether any contribution from joint absences. The species reduction therefore has only minor effect on the MDS; Fig. 3a is very similar to Fig. 1c.

The most notable feature of Fig. 3 is the clearer separation of the high dose replicates in the PCA configuration than for MDS and DECORANA. For nematodes, ANOVA tests of treatment differences on the the first 2 PC axes (each at the $p<0.025$ level) are marginally significant; the principal difference is between $\mathrm{H}$ and the other treatments. In a MANOVAbased analysis on the furst 5 PCs (which account for $72 \%$ of the total variance) there is a highly significant difference among treatments, and Malhanobis' distance tests show that this is again due to the contrast of $\mathrm{H}$ with the rest. For copepods, a similar pattern

\section{NEMATODES}
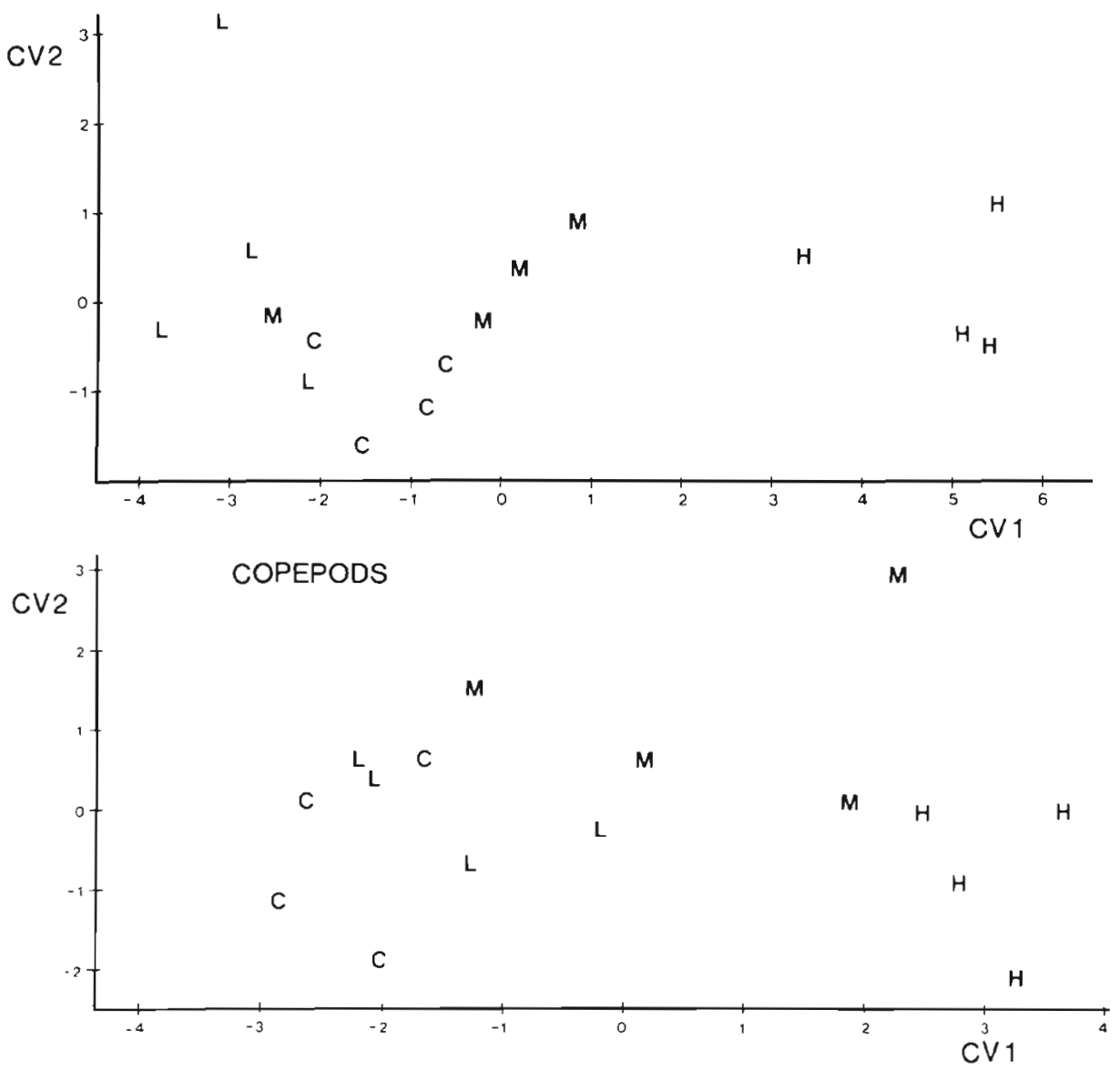

Fig. 4. Plot of Canonical variate 2 (CV2) versus Canonicai variate 1 (CV1) for the reduced species set of nematodes and copepods Treatment notation as Fig. 1 Only CV1 should be interpreted because only one $C V$ axis is hypothesized, and only one is statistically significant 
emerges: treatments are separated by an ANOVA on $\mathrm{PC} 2(p=0.02)$ and, while it is again mostly $\mathrm{H}$ versus the rest, here the treatment level means are in the sequence C-L-M-H. Again there is a highly significant difference among treatments in the MANOVA and Malhanobis' distances differ significantly between $\mathrm{H}$ and $\mathrm{L}, \mathrm{H}$ and $\mathrm{C}$, and $\mathrm{M}$ and $\mathrm{C}$.

When the overall MANOVA test (Roy's greatest root criterion, Seber 1984) is significant, it can be instructive to compare the order of treatment means on the first canonical variate CV1 (Seber 1984), which is the only MANOVA axis on which differences are expected here. Fig. 4 plots the first 2 canonical variates, both for nematodes and copepods; for the latter, CV1 is again in the sequence $\mathrm{C}-\mathrm{L}-\mathrm{M}-\mathrm{H}$. Such a monotone sequence of treatment levels has a probability of 1 in 12 (note that the direction of the CV1 axis is arbitrary). This gives a further test of between-treatment differences, which makes no parametric assumptions of normality and 'large-samples', unlike the MANOVA test. However, this simple non-parametric test is only capable of significance (as here) at the $p<0.10$ level.

It is important to note that Fig. 4 is not an ordination. Unlike MDS, PCA and DECORANA (Fig. 3), the CV plot uses the known division of the 16 samples into 4 replicates from 4 treatments (though not the known ordering of treatment severity). In fact, its rationale is to find the directions in the multi-dimensional species space that maximise the differences among the treatment means; thus a purely random data set would exhibit some separation on a CV1 axis. (A simple analogy here is with 'snooping' in the original species matrix, to summarise the data by one species, that whose abundance changes most between treatments; though this can sometimes be informative, its dangers are obvious.) The demonstration of treatment differ-

Table 1. Univariate measures for mesocosm nematodes and copepods. $I$ : total number of individuals per sample; $S$ : total number of species; $D$ : species richness; $H^{\prime}$ : Shannon-Wiener diversity index; $J$ : evenness; $N$ and $N^{\prime}$ : Hill's (1973) diversity and evenness numbers

\begin{tabular}{|c|c|c|c|c|c|c|c|c|c|c|c|c|}
\hline Basin & $I$ & $S$ & $D$ & $H^{\prime}$ & $J$ & $N_{1}$ & $N_{2}$ & $N_{\infty}$ & $N_{10}$ & $N_{10}$ & $N_{21}$ & $N_{21}^{\prime}$ \\
\hline \multicolumn{13}{|c|}{ Nematodes } \\
\hline $\mathrm{C}$ & 1560 & 26 & 5.42 & 2.73 & 0.83 & 15.4 & 11.9 & 17.0 & 0.59 & 0.57 & 0.77 & 0.75 \\
\hline$C$ & 1296 & 24 & 4.99 & 2.38 & 0.75 & 10.8 & 6.8 & 32.0 & 0.45 & 0.42 & 0.63 & 0.59 \\
\hline $\mathrm{C}$ & 864 & 35 & 7.39 & 3.28 & 0.92 & 26.7 & 26.4 & 8.1 & 0.76 & 0.75 & 1.06 & 1.06 \\
\hline C & 1620 & 30 & 6.31 & 2.96 & 0.87 & 19.4 & 16.3 & 13.1 & 0.64 & 0.63 & 0.84 & 0.83 \\
\hline L & 1920 & 32 & 6.70 & 3.11 & 0.89 & 22.5 & 20.3 & 13.7 & 0.70 & 0.69 & 0.90 & 0.89 \\
\hline L & 3840 & 26 & 5.44 & 2.81 & 0.86 & 16.7 & 13.4 & 16.2 & 0.64 & 0.62 & 0.80 & 0.79 \\
\hline L & 1416 & 34 & 7.16 & 3.08 & 0.87 & 21.8 & 14.9 & 22.0 & 0.64 & 0.63 & 0.68 & 0.66 \\
\hline $\mathrm{L}$ & 2544 & 25 & 5.21 & 2.60 & 0.80 & 13.5 & 9.6 & 23.0 & 0.54 & 0.52 & 0.70 & 0.68 \\
\hline$M$ & 1452 & 24 & 4.99 & 2.74 & 0.86 & 15.6 & 12.3 & 20.0 & 0.64 & 0.63 & 0.78 & 0.77 \\
\hline $\mathrm{M}$ & 2844 & 25 & 5.21 & 2.74 & 0.85 & 15.6 & 11.4 & 23.0 & 0.62 & 0.60 & 0.73 & 0.71 \\
\hline $\mathrm{M}$ & 1368 & 30 & 6.29 & 3.01 & 0.88 & 20.3 & 17.0 & 14.0 & 0.67 & 0.66 & 0.83 & 0.82 \\
\hline $\mathrm{M}$ & 1056 & 24 & 4.99 & 2.56 & 0.80 & 13.1 & 9.5 & 23.0 & 0.54 & 0.52 & 0.72 & 0.70 \\
\hline $\mathrm{H}$ & 2988 & 30 & 6.29 & 2.98 & 0.87 & 19.7 & 17.1 & 12.0 & 0.65 & 0.64 & 0.86 & 0.85 \\
\hline $\mathrm{H}$ & 3920 & 20 & 4.12 & 1.42 & 0.47 & 4.1 & 2.0 & 71.0 & 0.20 & 0.16 & 0.47 & 0.31 \\
\hline $\mathrm{H}$ & 3024 & 28 & 5.86 & 2.82 & 0.84 & 16.8 & 13.6 & 16.0 & 0.60 & 0.58 & 0.80 & 0.79 \\
\hline $\mathrm{H}$ & 3504 & 30 & 6.29 & 2.86 & 0.84 & 17.5 & 12.4 & 22.0 & 0.58 & 0.56 & 0.70 & 0.69 \\
\hline \multicolumn{13}{|c|}{ Copepods } \\
\hline$C^{*}$ & 70 & 21 & 4.79 & 2.70 & 0.88 & 14.9 & 13.4 & 20.0 & 0.71 & 0.69 & 0.89 & 0.89 \\
\hline C & 52 & 18 & 4.54 & 2.63 & 0.91 & 14.0 & 15.7 & 14.3 & 0.77 & 0.76 & 1.12 & 1.13 \\
\hline $\mathrm{C}$ & 125 & 23 & 4.63 & 2.67 & 0.85 & 14.6 & 12.7 & 13.0 & 0.63 & 0.61 & 0.87 & 0.86 \\
\hline C & 39 & 15 & 3.84 & 2.34 & 0.86 & 10.4 & 9.0 & 28.9 & 0.69 & 0.67 & 0.86 & 0.84 \\
\hline $\mathrm{L}$ & 54 & 19 & 4.60 & 2.65 & 0.90 & 14.3 & 14.4 & 18.0 & 0.75 & 0.73 & 1.00 & 1.01 \\
\hline $\mathrm{L}$ & 54 & 17 & 4.11 & 2.30 & 0.81 & 10.0 & 6.6 & 36.7 & 0.59 & 0.56 & 0.65 & 0.61 \\
\hline L & 93 & 21 & 4.44 & 2.75 & 0.90 & 15.8 & 15.2 & 13.3 & 0.75 & 0.73 & 0.96 & 0.96 \\
\hline $\mathrm{L}$ & 51 & 11 & 2.55 & 1.45 & 0.60 & 4.3 & 2.5 & 62.0 & 0.39 & 0.32 & 0.59 & 0.46 \\
\hline $\mathrm{M}$ & 70 & 15 & 3.35 & 2.08 & 0.76 & 8.0 & 4.9 & 43.1 & 0.53 & 0.50 & 0.60 & 0.55 \\
\hline $\mathrm{M}$ & 60 & 15 & 3.46 & 2.10 & 0.77 & 8.2 & 5.5 & 38.6 & 0.54 & 0.51 & 0.67 & 0.63 \\
\hline$M$ & 42 & 17 & 4.36 & 2.47 & 0.87 & 11.8 & 9.9 & 28.2 & 0.69 & 0.67 & 0.83 & 0.82 \\
\hline $\mathrm{M}$ & 93 & 16 & 3.33 & 1.89 & 0.68 & 6.7 & 3.6 & 51.1 & 0.41 & 0.37 & 0.54 & 0.45 \\
\hline $\mathrm{H}$ & 126 & 21 & 4.20 & 2.38 & 0.78 & 10.8 & 6.1 & 37.6 & 0.51 & 0.49 & 0.56 & 0.51 \\
\hline $\mathrm{H}$ & 374 & 26 & 4.25 & 1.21 & 0.37 & 3.4 & 1.8 & 74.5 & 0.13 & 0.09 & 0.52 & 0.32 \\
\hline $\mathrm{H}$ & 178 & 24 & 4.52 & 2.61 & 0.82 & 12.6 & 9.4 & 21.1 & 0.56 & 0.54 & 0.69 & 0.66 \\
\hline $\mathrm{H}$ & 168 & 25 & 4.74 & 2.19 & 0.68 & 9.0 & 4.5 & 44.3 & 0.36 & 0.33 & 0.50 & 0.44 \\
\hline
\end{tabular}


ences is in the MANOVA test; if that test is significant then the CV plot can help in assessment of where the differences are.

\section{Univariate analyses}

Values of diversity, evenness and species richness for the 16 replicate samples are given in Table 1 . To examine whether there are clear differences between treatments in the combination of the 12 univariate measures in this table, we have subjected combinations of these measures to Principal Components Analyses, and assessed the significance of any differences using ANOSIM. For both rematodes and copepods 3 separate analyses were done using: (a) all 12 measures, (b) omitting total numbers of individuals $(I)$, which as shown below are significantly higher in the high dose boxes, and (c) as (a) but excluding Hill's diversity numbers $\left(N\right.$ and $\left.N^{\prime}\right)$. For nematodes there are no significant differences at the $p<0.05$ level between treatments in any of these analyses. For copepods there are global differences at $p<0.05$. Fig. 5 shows the PCA analysis for subset (b) of the copepod data. The other 2 analyses (a) and (c) were virtually identical to this, and show that the differences between treatments in the univariate measures result from the difference between the high dose boxes and the others. As Gray et al (1988) found, there is effectively no additional information in the Hill's diversity numbers not already present in $S, D, H^{\prime}$ and $J$.

To construct $k$-dominance curves, data for the 4 replicates of each treatment have been combined. For

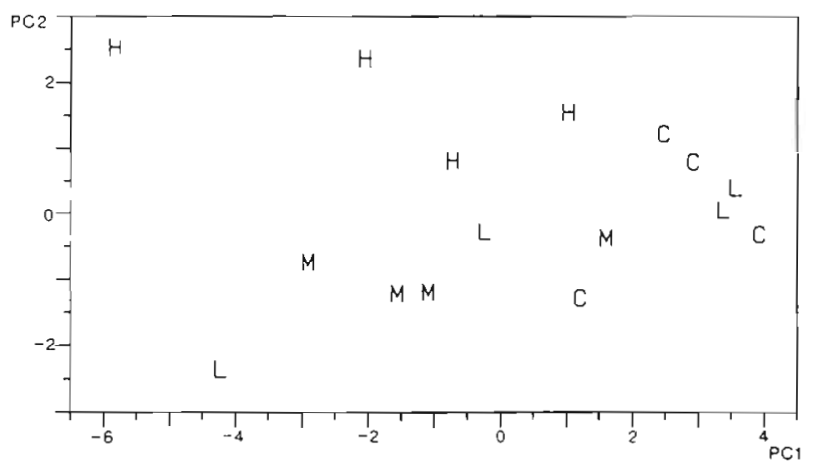

Fig. 5. PCA analysis of the univariate measures for copepods given in Table 1, excluding total number of individuals $(I)$. Treatment notation as Fig. 1. First and second PCs explain 77.5 and $17.0 \%$ of the variability respectively

nematodes (Fig. 6a) the curves for the 4 treatments lie very close together, and cross one another, indicating no clear-cut treatment effect on the diversity profile. For copepods, however, the high-dose $(H)$ and medium-dose $(\mathrm{M})$ treatment curves are clearly above the low-dose (L) and control (C) curves throughout their length, indicating lower diversity in the former (Fig. 6b). Although both the $H$ and $M$ and the $L$ and $C$ curves cross each other, this is only at the upper ends of the curves (rarer species), the lower ends forming a sequence from high to low diversity C-L-M-H. The chances of this sequence occurring by chance (in a direction predicted a priori) are 1 in 24 .

Comparison of the $k$-dominance curves for nematodes and copepods within treatments shows that for high- and medium-dose treatments (Fig. $7 a, b$ ) the copepod curve is well above the nematode curve

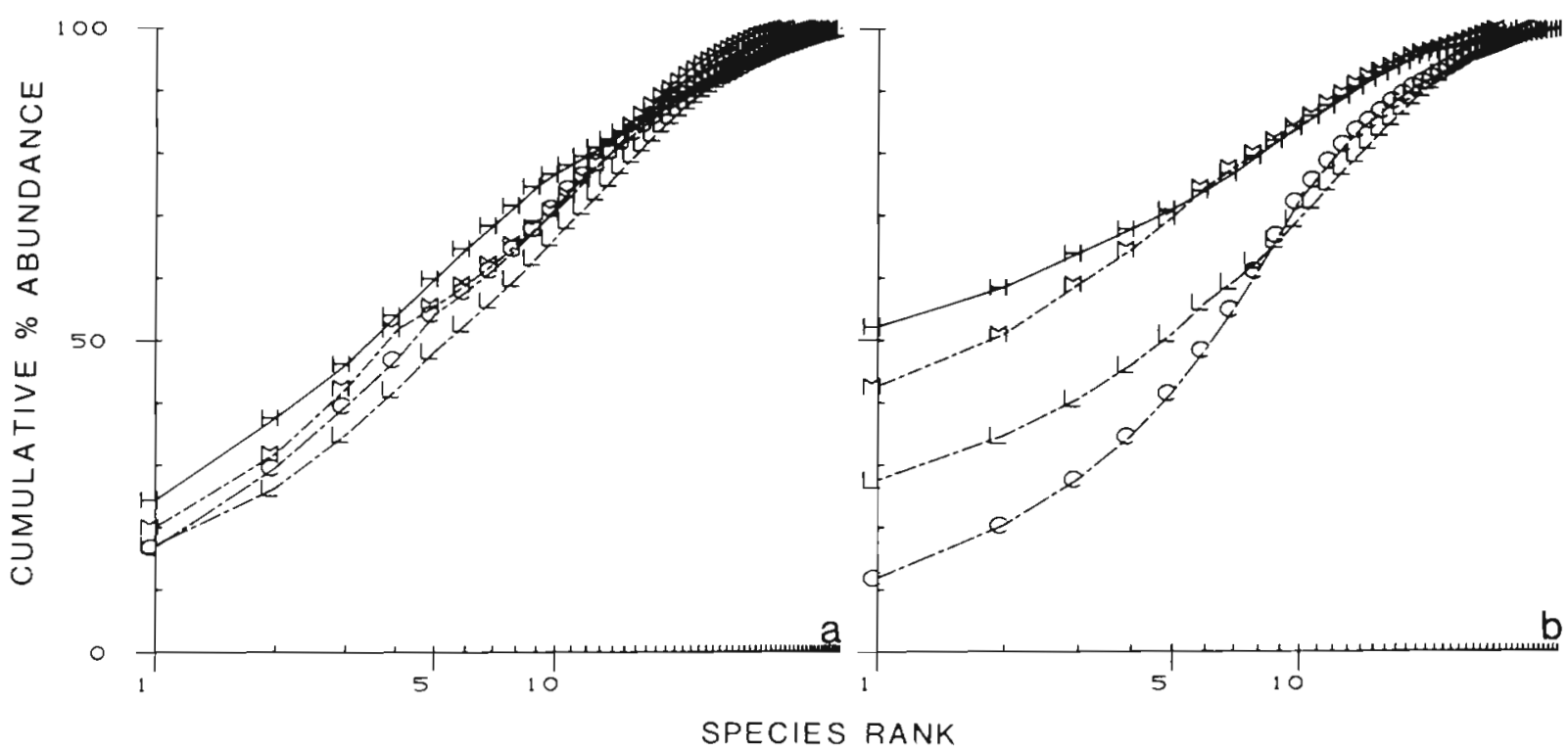

Fig. 6. $k$-dominance curves for (a) nematodes and (b) copepods; all replicates within treatments combined. Treatment notation as Fig. 1 


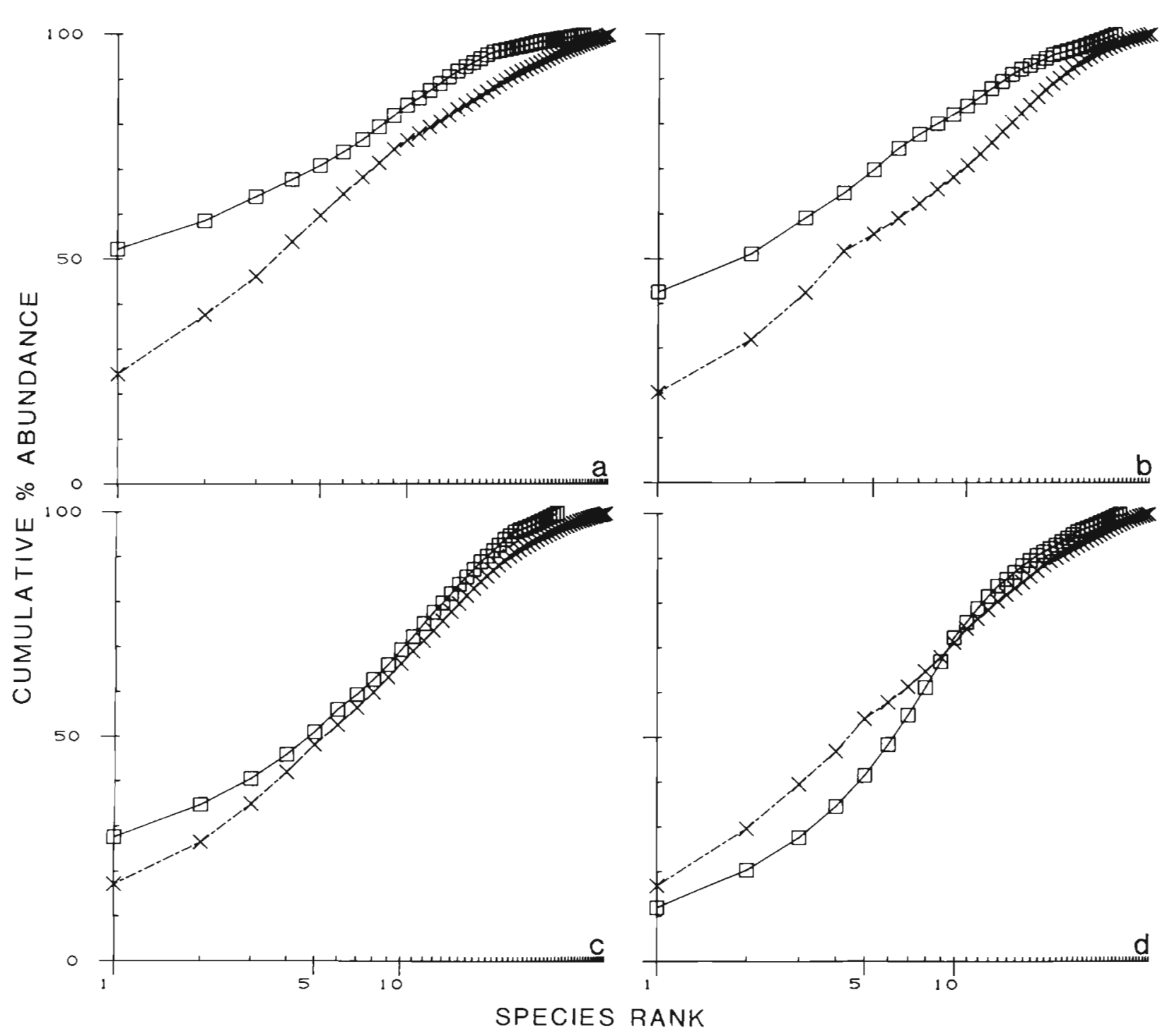

Fig. 7. Comparison of $k$-dominance curves for nematodes (crosses and broken lines) and copepods (squares and solid lines), for combined replicates within each treatment: (a) high dose, (b) medium dose, (c) low dose, (d) control

throughout its length, indicating lower copepod than nematode diversity. For the low-dose treatment (Fig. $7 \mathrm{c}$ ) the curves are close together and for the control (Fig. 7d) they cross, indicating no unequivocal separation in terms of diversity profiles. Despite this differential response in community structure between the nematode and copepod components of the meiobenthos, Table 2 shows clearly that there are no differences in the nematode:copepod ratio between treatments. However, there are differences in absolute abundance of both nematodes and copepods $(p<0.01$ in an ANOVA); for copepods these differences are entirely due to higher abundance in the high dose boxes, while for nematodes the high dose boxes are significantly higher than the medium dose and control, but not than the low dose.

\section{DISCUSSION}

In many respects, the various multivariate analyses give similar results. For nematodes, ANOSIM shows only significant differences between the high-dose $(\mathrm{H})$ and the medium-dose (M) and control (C) treatments. Malhanobis' distances are also only significant between $\mathrm{H}$ and the other treatments. For copepods, ANOSIM on the square root transformed data matrix shows significant differences between $\mathrm{H}$ and $\mathrm{M}, \mathrm{H}$ and $\mathrm{C}$, and $\mathrm{M}$ and $C$, whereas the Malhanobis' distances differ significantly between $\mathrm{H}$ and $\mathrm{L}, \mathrm{H}$ and $\mathrm{C}$ and $\mathrm{M}$ and $\mathrm{C}$.

However, comparable ordinations such as PCA, MDS and DECORANA lead to rather different configurations (Fig. 3), most obviously in their separation of $\mathrm{H}$ replicates from other treatments. The reason for this 
Table 2. Mesocosm meiofauna. Numbers of nematodes and copepods (per $17.9 \mathrm{~cm}^{2}$ of surface) and nematode to copepod ratios $(N: C)$

\begin{tabular}{|crrr|}
\hline Basin & Nematodes & Copepods & N:C \\
\hline C & 1560 & 70 & 22.3 \\
C & 1296 & 52 & 24.9 \\
C & 864 & 125 & 6.9 \\
C & 1620 & 39 & 41.5 \\
L & 1920 & 54 & 35.6 \\
L & 3840 & 54 & 71.1 \\
L & 1416 & 93 & 15.2 \\
L & 2544 & 51 & 49.9 \\
M & 1452 & 70 & 20.7 \\
M & 2844 & 60 & 47.4 \\
M & 1368 & 42 & 35.6 \\
M & 1056 & 93 & 11.4 \\
H & 2988 & 126 & 23.7 \\
H & 3920 & 374 & 10.5 \\
H & 3024 & 178 & 17.0 \\
H & 3504 & 168 & 20.9 \\
\hline
\end{tabular}

seems to lie in the poor 2-D representations of the full structure in the high-dimensional abundance matrix, particularly for PCA where the first 2 PCs account for $<40 \%$ of the total variability. This becomes most apparent when cluster analyses are performed, in the original full-dimensional space, and the clusters obtained at a range of similarity levels superimposed on the ordinations (e.g. Field et al. 1982). Dendrograms from hierarchical, group-average clustering are given in Fig. 8, based on $\log (1+x)$ transformed data and the reduced species set, for both Bray-Curtis (BC) similarities and the 'Euclidean distances' between replicates, the latter being the dissimilarity matrix implicit in a PCA. Cluster analysis groups together replicates which are in close proximity in the fulldimensional species space, so an acceptable 2-D ordination should preserve these groupings without distortion. Fig. $8 \mathrm{c}$ shows that, in spite of the relatively high stress, BC-based MDS and BC-based cluster analysis are in reasonable agreement. By contrast (Fig. 8f), Euclid-based PCA and Euclid-based clustering show considerable distortion with, for example, the $\mathrm{H} 4$ replicate grouped with $\mathrm{M} 2$ rather than $\mathrm{H} 1$ and $\mathrm{H} 3$. It is also interesting to note the complementary operations in Fig. $8 \mathrm{~d}$ and e, with the BC-based MDS reflecting the results of a Euclid clustering much more accurately than the Euclid-based PCA represents the BC clustering. Superimposing the 2 sets of cluster analysis groupings onto the DECORANA configuration produced a highly convoluted picture in both cases, indicating a poor 2-D representation of $\mathrm{BC}$ and Euclidean distance matrices.

So, the apparent discrepancies between, for exam- ple, Fig. $3 \mathrm{a}$ and $\mathrm{c}$ are a reflection of the different construction of the 2 techniques. In the 2-D PCA the third and higher PC components are ignored, as the points are projected perpendicularly onto the 'best' $2-\mathrm{D}$ plane, possibly on top of each other from 'opposite sides', as happens here with some of the $H$ replicates. This is less likely to happen in MDS, where an attempt is made to preserve the between-replicate dissimilarities from the full-dimensional space. Though some distortion (high 'stress') may still result, an MDS ordination is likely to offer a more realistic 2-D representation than a PCA.

The univariate indices and $k$-dominance plots indicate that the nematode diversity profiles are very similar between treatments, but for the copepods there is a clear treatment effect. The differences in copepod diversity profiles are mainly due to changes in the more abundant species; this is indicated both by the $k$ dominance plots and by comparison of ANOSIM results using square and 4 th root transformed abundance data. Indeed, the sequence C-L-M-H in the $k$ dominance plots is almost entirely due to increasing numbers of Tisbe species with increasing dose level. In the control treatment the nematode and copepod diversity profiles are not distinguishable from each other, but copepod diversity gradually decreases relative to nematode diversity as the pollution level increases. This differential response of nematodes and copepods was also found by Gee et al. (1985) in experiments in which the same meiobenthic assemblage from Bjørnehodet Bay was subjected to different levels of particulate organic input (their Fig. 2). Not only are these results quantitatively similar, but the most significant differences in the copepod assemblages between treatments in the organic enrichment experiment involved increases in Tisbe species, similar to those in the present experiment (Appendix 3, Table 9). There is no falloff in the number of copepod species present from the control to high dose treatments, and decreases in diversity result from disproportionate increases in abundance of a few species, principally Tisbe species but also Danielssenia typica and Diosaccid species 1 . These increases result in the significantly higher total abundance of copepods in the high dose boxes compared with the others. Tisbe species are known to have opportunistic characteristics, but Gee et al. (1985) argued that their increase in abundance under organic enrichment was not an experimental mesocosm artifact, and the same arguments apply to the present experiment

Tisbe species became paticularly abundant in the second replicate from the high dose basin. In this box the nematode Monhystera disjuncta also became overwhelmingly dominant, and this species was generally more abundant in all the high dose boxes than in the 

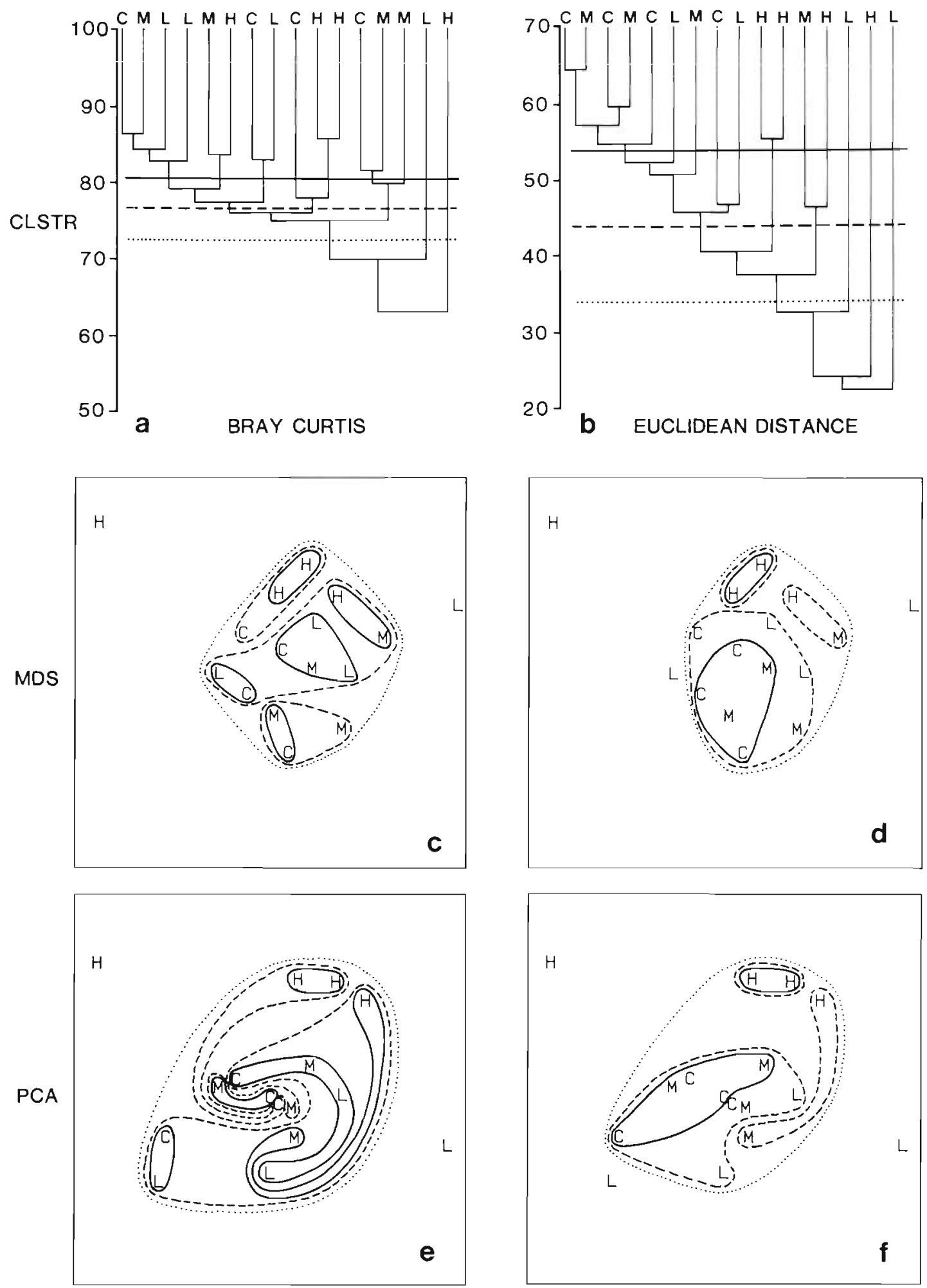

Fig. 8. (a) Cluster analysis of the reduced nematode data set using $\log (1+x)$ transformed species abundances, Bray-Curtis similarity measures and group average sorting. Treatment notation as Fig. 1. Solid, broken and dotted horizontal lines indicate levels of similarity arbitrarily selected to separate groupings which describe the major structure of the dendrogram. (b) as (a) but using the Euclidean distance matrix rather than Bray-Curtis. (c) MDS ordination from Fig. 3a with groupings from dendrogram (a) superimposed. (d) MDS ordination from Fig. 3a with groupings from dendrogram (b) superimposed. (e) PCA ordination from Fig. $3 c$ with groupings from dendrogram (a) superimposed. (f) PCA ordination from Fig. 3c with groupings from dendrogram (b) superimposed 
others. Its ease of laboratory culture and very fast turnover time (Vranken \& Heip 1986) suggest that this species too can be regarded as relatively opportunistic. Its high abundance in the second $\mathrm{H}$ replicate is largely responsible for the relative isolation of this sample on the MDS configurations (Fig. 1).

The above responses of the meiobenthic community clearly result from additions of different levels of hydrocarbons and copper, but at least 3 possible mechanistic explanations for the pollution reponse can be invoked.

(1) Copepods are generally more sensitive to pollution than nematodes; this results in a decrease in their diversity relative to that of the nematodes with increasing levels of pollution.

(2) The levels of exposure to pollutants differed for the 2 groups. Measured levels of hydrocarbons and copper in the sediments were not significantly different between treatments (Gray et al. 1988), whereas concentrations in water showed a clear dosing gradient across basins, so the differential response might result from the fact that copepods are mainly epibenthic and nematodes mainly burrowing.

(3) The general response was to organic enrichment brought about by the addition of hydrocarbons and/or by mortalities of large macrobenthic species. Observed responses could be explained by the fact that certain opportunistic copepods, particularly Tisbe species, had higher colonising potential than any of the nematode species.

The general similarity of the response to that of nutrient enrichment, and the fact that changes in copepod community structure resulted mainly from selective increases of certain species rather than selective mortalities, favour the third explanation. Rates of population increase for Tisbe species at temperatures as low as those in the mesocosm experiment $\left(9^{\circ} \mathrm{C}\right)$ are not known, but certainly Tisbe cultured at higher temperatures have rates of increase higher than those for most other harpacticoid taxa (Hicks \& Coull 1983, Table VI). The nematode Monhystera disjuncta has a generation time of $18 \mathrm{~d}$ at $9^{\circ} \mathrm{C}$ i this is very much shorter than for any other nematode species studied, at comparable temperatures, by Vranken \& Heip (1986). This species could potentially have passed through 4 generations during the experiment, whereas the other species studied by Vranken \& Heip could barely have completed 1 generation. It therefore seems that the observed reponse of the meiofauna community results from increases in populations of those species of copepods and nematodes which have the capacity to do so over the time-scale of the experiment. Toxicity of the pollutants was not an important element of the response, but may have had an effect if the experiment. had been run for longer or if the pollutants had penetrated into the sediments.
Acknowiedgements. We are grateful to Helen Archer and John Hall for computing assistance.

\section{LITERATURE CITED}

Bakke, T., Follum, O. A., Moe, K. A., Sørensen, K. (1988). The GEEP Workshop: mesocosm exposures. Mar. Ecol. Prog Ser. $46: 13-18$

Bell, S. S., Coull, B. C. (1978). Field evidence that shrimp predation regulates meiofauna. Oecologia (Berl.) 35: $141-148$

Boucher, G. (1979). Evolution des caracteristiques chimiques et biologiques des sediments en circuit clos. 2. Effetes de la matiere organique circulante sur la meiofaune de systems polytrophes. Publ. Sci. Tech. CNEXO: Actes calloq. 7 : $31-48$

Boucher, G., Chamroux, S. (1976). Bacteria and meiofauna in an experimental sand ecosystem. 1. Material and preliminary results. J. exp. mar. Biol. Ecol. 24: 237-249

Cantelmo, F. R., Rao, K. R. (1978). Effect of pentachlorophenol (PCP) on meiobenthic communities established in an experimental system. Mar. Biol. 46: 17-22

Cantelmo, F. R., Tagatz, M. E., Rao, K. R. (1979). Effect of barite on meiofauna in a flow-through experimental system. Mar. environ. Res. 2: 301-309

Chamroux, S., Boucher, G., Bodin, P. (1977). Etude experimentale d'un ecosystem sableux. 2. Evolution des populations de bacteries et de meiofaune. Helgoländer wiss. Meeresunters. 30: 163-177

Clarke, K. R., Green. R. H. (1988). Statistical design and analysis for a 'biological effects' study. Mar. Ecol. Prog. Ser. 46: 213-226

Elmgren, R., Frithsen, J. B. (1982). The use of experimental ecosystems for evaluating the impact of pollutants: a comparison of an oil spill in the Baltic Sea and two long term, low level oil additions in mesocosms. In: Grice, G. D., Reeve, M. R. (eds.) Marine mesocosms. Springer Verlag, New York, p. 153-165

Elmgren, R., Vargo, G. A., Grassle, J. F., Grassle, J. P., Heinle, D. R., Langlois, G., Vargo, S. L. (1980). Trophic interactions in experimental marine ecosystems perturbed by oil. In: Giesy, J. P. (eds.) Microcosms in ecological research. National Technical Information Service, Springfield, Va., p. $779-800$

Field, J. G., Clarke, K. R., Warwick, R. M. (1982). A practical strategy for analysing multispecies distribution patterns. Mar. Ecol. Prog. Ser. 8: 37-52

Gee, J. M., Warwick, R. M., Schaanning, M., Berge, J. A., Ambrose, W. G. (1985). Effects of organic enrichment on meiofaunal abundance and community structure in sublittoral soft sediments. J. exp. mar. Biol. Ecol. 91: 247-262

Grassle, J. F., Elmgren, R., Grassle, J. P. (1980). Response of benthic communities in MERL ecosystems to low level chronic additions of No. 2 fuel oil. Mar. environ. Res. 4: $279-297$

Gray, J. S., Aschan, M., Carr, M. R., Clarke, K. R., Green, R. H., Pearson, T H., Rosenberg R., Warwick, R. M. (1988). Analysis of community attributes of the benthic macrofauna of Frierfjord/Langesundfjord, and in a mesocosm experiment. Mar. Ecol. Prog. Ser. 46: 151-165

Heip, C., Warwick, R. M., Carr, M. R., Herman, P. M. J., Huys, R. Smol N., Van Holsbeke, K. (1988). Analysis of community attributes of the benthic meiofauna of Frierfjord/ Langesundfjord. Mar. Ecol. Prog. Ser. 46: 171-180

Hicks, G. R. F., Coull, B. C. (1983). The ecology of marine 
meiobenthic harpacticoid copepods. Oceanogr mar. Biol. A. Rev. 21:67-175

Hill. M. O. (1973). Diversity and evenness: a unifying notation and its consequences. Ecology 54: 427-432

Hill, M. O., Gauch, H. G. (1980). Detrended correspondence analysis, an improved ordination technique. Vegetatio 42 : $47-58$

Lambshead, P. J. D., Platt, H. M., Shaw, K. M. (1983). The detection of differences among assemblages of marine benthic species based on an assessment of dominance and diversity. J. nat. Hist. 17. 859-874

McIntyre, A. D. (1969). Ecology of marine meiobenthos. Biol. Rev. 44: 245-290

McIntyre, A. D., Munro, A. L. S., Steele, J. H. (1970). Energy flow in a sand ecosystem. In: Steele, J. H. (ed.) Marine food chains. Oliver \& Boyd, Edinburgh, p. 19-31

McIntyre, A. D., Warwick, R. M. (1984). Meiofauna techniques. In: Holme, N. A., McIntyre, A. D. (eds.) Methods for the study of marine benthos. Blackwell, Oxford, p. $217-244$
McLachlan, A., Dye, A., Harty, B. (1981). Simulation of the interstitial system of exposed sandy beaches. Estuar. coast. Shelf Sci. 12: $267-278$

Raffaelli, D., Mason, C. F. (1981). Pollution monitoring with meiofauna using the ratio of nematodes to copepods. Mar Pollut. Bull. 12: 158-163

SAS User's Guide, Version 5 (1985). SAS Institute Inc., Cary, North Carolina

Seber, G. A. F. (1984). Multivariate observations. Wiley, New York

Vranken, G., Heip, C. (1986). The productivity of marine nematodes. Ophelia 26: 429-442

Warwick, R. M. Gee, J. M., Berge, J. A., Ambrose, W. (1986). Effects of the feeding activity of the polychaete Streblosoma bairdi (Malmgren) on meiofaunal abundance and community structure. Sarsia 71: 11-16

Wormald, A. P., Stirling, H. P. (1979). A preliminary investigation of nutrient enrichment in experimental sand columns and its effect on tropical intertidal bacteria and meiofauna. Estuar. coast. mar. Sci. 8: 441-453 